\title{
Interfacial fracture between highly crosslinked polymer networks and a solid surface: Effect of interfacial bond density
}

\author{
Mark J. Stevens \\ P.O. Box 5800, MS 1111, Sandia National Laboratories, Albuquerque, NM 87185-1111 \\ (March 7, 2000)

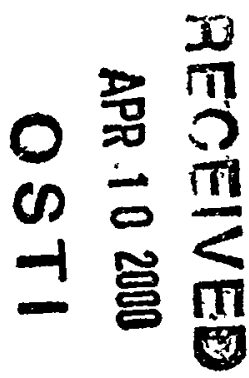 \\ For highly crosslinked, polymer networks bonded to a solid surface, the effect
} of interfacial bond density as well as system size on interfacial fracture is studied molecular dynamics simulations. The correspondence between the stress-strain curve and the sequence of molecular deformations is obtained. . The failure strain for a fully bonded surface is equal to the strain necessary to make taut the average minimal path through the network from the bottom solid surface to the top surface. At bond coverages less than full, nanometer.. scale cavities form at the surface yielding an inhomogeneous strain profile. The failure strain and stress are linearly proportional to the number of bonds at the interface unless the number of bonds is so few that van der Waals interactions dominate. The failure is always interfacial due to fewer bonds at the interface than in the bulk.

\section{INTRODUCTION}

Adhesives present a complex problem for polymer theory. Progress has primarily occurred for adhesives with weak interfacial strength [1] such as pressure sensitive adhesives [2] and polymer-polymer interfaces [3]. Such adhesives typically do not have strong chemical bonds across the interface. Only relatively weak van der Waals interactions exist at the interace. For strong adhesives equivalent development is lacking. The difficulty of performing 
measurements on interfaces is a major obstacle in understanding adhesion problems. Recent experiments at Sandia have begun to study failure in the strong interface of an epoxy on a silicon wafer $[4,5]$. The interfacial structure is probed with neutron and X-ray reflectivity. In addition, the number of chemical bonds at the interface is varied in a controlled manner through use of self-assembled monolayer (SAM) coatings. In conjunction with these experiments, a program of molecular dynamics (MD) simulations have been developed and performed. The initial results of these MD simulations are reported here.

In bulk epoxies, experiments have shown that as the crosslink density increases the bulk failure mechanism changes from crazes to deformation zones [6]. There is very little information about mechanisms for interfacial failure in epoxies on solid surfaces. Theoretically, linear elastic fracture mechanics works well for such polymers far from the crack tip. However, the method breaks down near the crack tip where large plastic deformation occurs and the molecular details become important [7]. Performing MD simulations that treat the region near the crack tip would yield very useful, but missing information.

From a general perspective, the polymer adhesive is a network. We would like to know how the network structure influences the adhesive strength, the initiation of cracks, the distribution of stress, etc. In a random network, the stress on bonds is expected to vary, and the network bonds that are most stressed will break first [8]. Crack initiation could occur where a cluster of highly constrained bonds exists. There is no reason to expect the crack initiation site to occur at the interface, although we know experimentally that interfaces are often weak.

Termonia has performed a several calculations focussed originally on the strength of fibers [9]. These calculations are based on a lattice model of the network. His work on network polymers has primarily treated bulk deformations in elastomers.

Little work exists for molecular simulation of polymer adhesives [10]. Highly crosslinked polymer networks been particularly neglected by simulations, yet they are important class of adhesives. The development of MD simulations has reached the point where treatment of adhesives is possible [10]. Simple coarse-grained bead-spring models successfully treat bulk 


\section{DISCLAIMER}

This report was prepared as an account of work sponsored by an agency of the United States Government. Neither the United States Government nor any agency thereof, nor any of their employees, make any warranty, express or implied, or assumes any legal liability or responsibility for the accuracy, completeness, or usefulness of any information, apparatus, product, or process disclosed, or represents that its use would not infringe privately owned rights. Reference herein to any specific commercial product, process, or service by trade name, trademark, manufacturer, or otherwise does not necessarily constitute or imply its endorsement, recommendation, or favoring by the United States Government or any agency thereof. The views and opinions of authors expressed herein do not necessarily state or reflect those of the United States Government or any agency thereof. 


\section{DISCLAIMER}

Portions of this document may be illegible in electronic image products. Images are produced from the best available original document. 
polymer melts and networks $[11,12]$. This work extends these methods to study fracture of highly crosslinked polymer networks. Connectivity is the main ingredient ingredient in polymer dynamics [13]. The bead-spring models preserve the network connectivity. In these models, a bead represents of group of monomers; how many monomers depends on the particular polymer. Typically, the stiffer the polymer, the more monomers per bead. The coarse-graining allows the simulations to reach times of at least a microsecond. This enables coarse-grained MD simulations to treat entanglement dynamics [14]. In contrast, atomistic simulations can barely pass a nanosecond. Much of the important dynamics in polymers, let alone in adhesion occurs on longer time scales.

In order to understand how varying interfacial bonding effects the interfacial strength, the number of chemical bonds between the adhesive and the solid surface is varied. The stress-strain curves are then calculated as a function the number of interfacial bonds using MD. The structural dynamics is examined and correlated with the stress-strain curves.

In the following section, the bead-spring model is described along with all the aspects of the simulations. The results of the simulations are presented in sec. III. The correspondence between the stress-strain curve and the sequence of molecular deformation is given. How the structure determines the failure strain is described. Insights based on force measurements between self-assembled monolayers reveal the expected range of stress magnitudes for each of the possible interaction types. Next, the data for the stress-strain curves as a function of the number of interfacial bonds are given. In sec. IV, the results are discussed. The inhomogeneity in the strain is demonstrated and shown to be an additional constraint on the network effecting the failure strain. Finally, the conclusions are given in sec. V. 


\section{SIMULATION METHOD}

\section{A: Polymer Model and Potentials}

The polymer network is treated as a bead-spring system. Beads interact via a LennardJones (LJ) potential with a cutoff at $2.5 d$ [12].

$$
U_{\mathrm{LJ}}(r)=4 u_{0}\left[\left(\frac{d}{r}\right)^{12}-\left(\frac{d}{r}\right)^{6}\right]
$$

In this article $d$ represents the LJ diameter and $u_{0}$ represents the LJ energy. The stress and strain will retain their traditional notation of $\sigma$ and $\varepsilon$, respectively. All quantities will be in LJ units. ,

The LJ stress will later be mapped to $\mathrm{MPa}$ to give a rough magnitude for the stresses. Because of the coarse-grained nature, there is no one-to-one mapping of the model to real systems. In coarse-grained simulations, the goal is to understand the model system selfconsistently. In relating the simulation results to actual adhesive such as epoxies, the emphasis is on the connectivity and its implications:

In the Kremer-Grest model [12], beads are bonded together using a potential that prevents chain crossing. This bond potential is the sum of the purely repulsive LJ interaction with a cutoff at $2^{1 / 6} d$ and a finite-extensible nonlinear elastic (FENE) attractive potential. In order to break bonds and preserve the continuity of the bond force, a breakable quartic bond potential was created to replace the FENE potential.

$$
U_{4}(r)= \begin{cases}k_{4}\left(r-r_{1}\right)\left(r-r_{2}\right)^{3}+U_{0}, & r<r_{2} \\ U_{0}, & r \geq r_{2}\end{cases}
$$

The potential parameters are: $k_{4}=1434.3 u_{0} / d^{4}, r_{1}=0.7411 d, r_{2}=0.0$ and $U_{0}=67.2234 u_{0}$. With this bond potential, chains can cross only by breaking bonds.

The maximum bond force is $156.7 u_{0} / d$, and the maximum LJ force is $2.4 u_{0} / d$. For atomic force-fields, the force ratio between the bond and the van der Waals forces is about 1000. However, a single bead represents several atoms and the LJ pair interaction represents 
multiple van der Waals pair interactions. With even just 3 atoms per bead, there are 9 pair interactions. A force ratio of order 100 in the coarse-grained model is representative of the atomic system. The ratio of 65 used in these simulations is then in the correct range.

\section{B. Solid Surface Model}

The complete system consists of a polymer network between two solid walls. Each wall is two layers of particles in an fcc lattice with nearest neighbor distance $1.204 d$. The (111) direction ( $z$-direction) is perpendicular to the walls. The wall particles are bound to the lattice sites by a harmonic spring with spring constant $100 u_{0} / d^{2}$. The wall dimensions give the simulation cell lateral lengths, $L_{x}$ and $L_{y}$, and periodic boundary conditions are applied in these directions. The separation distance between the innermost wall layers is $L_{z}$. The wall particles interact with the beads via the same LJ potential (Eq. 1), with some wall particles bonded to the polymer network by Eq. 2. To perform the tensile pull and shear simulations, the walls are pulled at constant velocity.

\section{Network Formation}

To form the networks, we take a cue from a highly crosslinked adhesive, epoxies. Epoxies are chemically cured networks formed from a liquid mixture of a resin (Bisphenol A) and a crosslinker [15]. Each strand consists of only a few monomers. As a bead corresponds typically to 2 or 3 monomers [12], an epoxy model would have only a few beads per strand. The minimal case of two beads is used here. In this initial work the emphasis is on understanding general features of highly crosslinked networks. In later work, the simple modifications that would better model epoxies, for example, will be applied. A discussion of possible modifications is given in sec IVC.

In epoxies, a liquid mixture of a crosslinker and a resin is crosslinked dynamically. In the simulations the mixture consists of two bead and three bead molecules. The three bead molecule has a sixfold functional crosslinker bead already bonded to a two bead strand. 
In this initial work, the strand beads and crosslinker beads have identical LJ parameters. The liquid is equilibrated at temperature $T=1.1 u_{0}$. After equilibrating the liquid mixture, crosslinkers are first bonded to the walls. Bonds are formed when the separation between a crosslinker and a strand end or wall particle is less than $1.3 \mathrm{~d}$. Next, during a MD run, the crosslinkers are bonded to strands until at least $95 \%$ of all possible bonds are made. Zero load is maintained on the walls during the crosslinking. Afterward, the temperature is reduced below the glass transition temperature $\left(T_{g}=0.5\right)$ to 0.3 .

\section{System parameters}

Four different system sizes have been studied. Table 1 lists the varying size parameters for these systems. For system 1 the wall dimensions are $L_{x}=33.1 d$ and $L_{y}=19.3 d$. For systems $2-4, L_{x}$ is doubled and $L_{y}=28.9 d$. Given the size of the epoxy molecules, the bead size corresponds to about $d \simeq 1 \mathrm{~nm}$. Using this mapping, the largest system studied has a height of $77 \mathrm{~nm}$. These are small systems compared to typical adhesives. The simulations effectively treat the region at or near the crack tip. In this region, strains and possibly even stresses are much larger than the macroscopic measured values.

The dynamics is performed at constant temperature $T$ using the Langevin thermostat [16]. The integration time step is $0.005 \tau$, and the damping constants are $1 \tau^{-1}$ for the monomers and $5 \tau^{-1}$ for the walls, where $\tau$ is the LJ time unit.

Simulations of both tensile and shear deformations are performed. In both cases, the walls are moved at constant velocity. All the data presented here is for a wall velocity of $v=0.01 d / \tau$. Simulations have been performed for $v=0.001 d / \tau$ and $0.1 d / \tau$ with similar results. For the shear simulations, the wall is pulled in the $x$-direction. 


\section{RESULTS}

\section{A. Molecular scale deformation of network}

Figure 1 shows a typical tensile stress-strain curve. The correspondence between the molecular deformations and the stress-strain curve is as follows. The first peak at $\varepsilon_{y}=0.1$ is the yield stress. This occurs due to the LJ force between pairs of neighboring beads going through its maximum value. In equilibrium, the typical separation is $1 d$, and the maximum force is at $r=2^{1 / 6} d=1.12 d$. Thus it takes a strain of about 0.1 to reach the maximum value.

At $\varepsilon>\varepsilon_{y}$ the stress is constant for a range of stains. In this plateau regime, examination of configurations shows that the bonds are not stretched. Instead the strands connecting two crosslinkers are pulled progressively taut. In this section, 'strand' includes the crosslinkers so that there are four beads to a strand. Only after the strands are in the taut, linear conformation do the bonds begin to stretch. Even though there are only four beads per strand, the strain to extend the strand from the compact initial state to the linear state is relatively large. For example, consider the close packed, planar state with the strand making a zig-zag path from one crosslinker to the other. The bond length is about equal to $d$ and in the compact state the nonbonded beads are separated by the core diameter, $d$. For this state the crosslinker-crosslinker separation distance is $\sqrt{3} d$. In the linear, taut conformation the separation is $3 d$. The strain to deform the compact state into the linear state is $\sqrt{3}$ ! The plateau regime in Fig. 1 has range of strain of about 0.35. Clearly, the average strand conformation is not in the close packed state at $\varepsilon=0$, but also it is clear that large strains are possible even for short strands.

Once strands are taut, bonds must stretch on further displacement. At this point, the stress rises. The points show the strains at which bonds break. The first bond breaking occurs at $\varepsilon=0.60$. As more bonds are stretched the stress rises with some bonds breaking. Finally, the system fails at $\varepsilon_{f}=1.05$, where the failure strain is taken as the stain at the 
midpoint between the failure stress and zero stress. Failure is interfacial with all the bonds between the bottom surface and the network breaking.

In all the simulations failure occurs interfacially. The reason for the interfacial failure is that the number of bonds at the interface is less than elsewhere. The crosslinkers bonded to the bottom surface have only a single bond in the $-z$ direction. Crosslinkers are allowed to bond to multiple wall sites, but they choose to bond to only a single site during the crosslinking procedure. Issues concerning this will be discussed in sec. IV. Crosslinkers not at the interface have close to 3 bonds in the $+z$ direction and $-z$ direction. Thus, the interfacial crosslinker bonds have to support more load, and they stretch first and break first.

\section{B. Minimal paths}

To understand the failure strains in terms of the network structure, we need to know what is the maximum strain possible without bonds breaking. It turns out that the average value of this maximum strain corresponds to the failure strain. An upper limit to the strain at which scission must occur is given by the minimal path lengths of the network. For a site on the bottom wall to which the network is bonded, there are many paths through the polymer network to the top wall. The shortest path is the minimal path $P$ for that site on the bottom wall. For the complete system there is a set of minimal paths, one for each bonding site at the bottom wall. The strain at which the strands in the minimal path are taut, but the bonds are not stretched is given by the relation,

$$
\varepsilon_{P}=\left(P-L_{z}\right) / L_{z}
$$

For $\varepsilon>\varepsilon_{P}$, some bond within the minimal path must stretch. Using Dijkstra's method [17], $P$ has been calculated for all bonding sites on the bottom wall. For the system in Fig. 1 the shortest minimal path has $\varepsilon_{P}=0.54$ which is slightly smaller than the first broken bond indicated by the points in the figure. Additional strain is required to stretch bonds 
to the breaking point. The simulations show that the average minimal path over the whole system is a good estimate of the failure strain. For the system in Fig. 1 the average value corresponds to $\varepsilon_{P}=0.97$ which is where the peak stress occurs.

The apparent large failure strain is now understood in terms of the physical constraints on the network connectivity. Bond breaking does not occur until the minimal paths in the network are taut. The strain to make the minimal paths taut is typically about 1 , since the typical conformation of a minimal path at $\varepsilon=0$ is a zig-zag path as one would expect. Bonds could break at small $\varepsilon$ if there were an additional constraint operating on a segment of the minimal paths. The fact that the failure strain corresponds to the minimal path strain shows that there are no additional constraints. However, for the systems with a low number of interfacial bonds, an additional constraint does arise and the failure strains are smaller than the minimal path strain. These results will be presented in sections IIIE and IIIF.

\section{Stress magnitude}

The number of bonds across the interface is an important quantity affecting interfacial fracture. The ideal stress to fracture the interface is directly proportional to the number of interfacial bonds, $N_{b}$.

$$
\sigma_{\text {id }}=N_{b} F_{b} / A \text {, }
$$

where $F_{b}$ is the force to break a single bond and $A$ is the area of the interface. For systems 2-4 where $N_{b} \simeq 200, F_{b}=156.7$ and $A=1928, \sigma_{\text {id }}=16.3$. This ideal value never occurs in the simulations, because the bonds are broken sequentially, not simultaneously (Fig. 1). Thus, at any given instant the contribution to the total stress by the breaking bonds is a fraction of the ideal value. The failure stress in Fig. 1 is $6.2 u_{0} / d^{3}$ which is 2.6 times smaller than the $\sigma_{\text {id. }}$.

One means to calibrate the simulations is comparison to measurements on self-assembled monolayers (SAMs). In addition, some fundamental aspects concerning the relative importance of van der Waals and chemical bonds at the interface are revealed. A SAM can be 
coated onto a force microscope tip, and the force to separate two SAMs can be measured. In this manner the molecular scale forces are measured which correspond to the stresses measured in the simulations. Thomas et al. [18] have measured methyl terminated alkylsiloxane SAMs as well as acid-base (COOH: $\mathrm{NH}_{2}$ ) terminated SAMs corresponding to the bonding of epoxies to silicon oxide surfaces. These experiments measure the pull-off force for a spherical tip of radius, $R$. To compare to the simulations the sphere-plane stress needs to converted to a plane-plane stress. For two surfaces interacting via a LJ potential, the repulsive core can be neglected. Writing the attractive part as $u(r) \stackrel{=}{=}-C / r^{6}$, then it can be shown [19] that the pull-off force between a sphere and a plane is

$$
F_{\mathrm{sp}}=\frac{\pi^{2} \rho^{2} C R}{6 D^{2}}
$$

where $\rho$ is the density and $D$ is the separation distance. Between two planes the pull-off force per area or stress is

$$
\sigma_{\mathrm{pp}}=\frac{\pi \rho^{2} C}{6 D^{3}}=\frac{F_{\mathrm{sp}}}{R} \frac{1}{\pi D}
$$

The experimental value for methyl terminated SAMs is $F_{\mathrm{sp}} / R=0.4 \pm 0.2 \mathrm{~N} / \mathrm{m}$ [18]. Taking $D$ as $4 \AA$, the typical LJ diameter for methyl groups, we obtain $\sigma_{\mathrm{pp}}=320 \pm 160 \mathrm{MPa}$. For the acid-base measurements, $F_{\mathrm{sp}} / R=4.3 \pm 0.2 \mathrm{~N} / \mathrm{m}$ which gives $\sigma_{\mathrm{pp}}=3400 \pm 320 \mathrm{MPa}$. However, the acid-base interaction does not have the LJ form. If we use the traditional JKR calculation, we obtain $3100 \pm 290 \mathrm{MPa}$. These two numbers are within the experimental uncertainty so that either number will satisfy the purposes of this discussion. The chemically bonded surfaces require a stress about 10 times larger than the van der Waals bonded surfaces. Moveover, both of these stresses are much larger than measured values for epoxy adhesives in mode II deformations $[5,20]$. While measuring the stress at a crack tip is not directly possible, estimates of crack tip stresses are an order of magnitude lower than the 3.4 GPa. This presents an apparent paradox concerning how bonded interfaces fail at much lower stresses.

The paradox is resolved by recognizing that the number of bonds at the interface for an epoxy adhesive is much smaller than for SAMs. The area per chain for the alkylsiloxane 
SAMs is about $25 \AA^{2}$. The area per crosslinker molecule is calculated using the bulk epoxy density, $1.13 \mathrm{~g} / \mathrm{cc}$ for the epoxy used in experiments at Sandia [15,4,21]. For a slab with thickness $8 \AA$ near a flat silicon oxide surface, the area per crosslinker is $920 \AA^{2}$. For the six-fold functional crosslinker at the interface assuming on average that 3 bonds are within the network and 3 bonds attach to the surface, then the area per interfacial bond is $310 \AA^{2}$. Thus, the area per bond in the epoxies is 12 times smaller than in the SAMs. This lowers the ideal fracture stress from $3400 \mathrm{MPa}$ to $280 \mathrm{MPa}$. However, with the small contact area of a force microscope the bonds between two SAMs can break almost simultaneously. In an epoxy, the bonds break successively, i.e. a crack propagates. The present simulations find that this reduces the failure stress by a factor of 2.6. Using this number the expected failure stress for epoxies on a silicon oxide surface under tensile strain is about $110 \mathrm{MPa}$. There are some uncertainties in this number. It is not likely that the crosslinker can make three bonds to the surface. Once one bond is made, the other bonds are restricted to occur in a small area about the first bond. On the other hand, the density at the surface is larger than in the bulk and this could easily compensate for the decreased bonding. Thus, including these uncertainties, the true number is not going to be far from $100 \mathrm{MPa}$. Finally, for epoxies not chemically bonded to a surface, but attached only by van der Waals interactions (i.e. epoxies on a SAM coated surface to prevent chemical bonding) the failure stress is $120 \mathrm{MPa}$, where the same 2.6 factor has been used. Within the uncertainties the contribution to the failure tensile stress due to chemical bonds and the contribution due to van der Waals interactions are equal. In addition, even the van der Waals stress is larger than the yield stress which is about $50 \mathrm{MPa}$.

The SAM systems can easily be modeled in the bead-spring system. To each of the top surface beads, a two bead SAM chain is bonded. Two such SAM coated surfaces are created. The stress to separate is calculated just as in the model network systems. The methyl terminated case corresponds to no bond between the two SAMs; the SAMs interact only through the LJ potential. In this case, the failure stress is $5.7 u_{0} / d^{3}$. This implies that 
$u_{0} / d^{3}=320 / 5.7=56 \mathrm{MPa}$. By bonding each of the two SAM chains opposite each other, the chemically bonded case is treated. In this case, the failure stress is $112 u_{0} / d^{3}$. This gives a map of $u_{0} / d^{3}=3400 / 112=30 \mathrm{MPa}$. Being a coarse-grained model, the two maps are not identical. An average value of $u_{0} / d^{3}=43 \mathrm{MPa}$ gives the best approximation.

The failure stress of the bead-spring network is $6.0 \mathrm{\epsilon} / \mathrm{d}^{3}$. This stress value is about equal to the nonbonded SAM failure stress. The above calculation from the experimental SAM data found that the epoxy failure stress would be about equal to the methyl terminated SAM failure stress. This agreement is a nice consistency.

\section{Variation of surface bond density}

Even though the actual value of the fracture stress is not equal to the ideal value, the failure stress may still scale with $N_{b}$. The dependence on $N_{b}$ can be examined in a controlled . manner by forbidding bonds to occur on a subset of the wall surface. Experimentally, this is done by coating the surface with SAMs which prevent chemical bonding $[5,20]$. In the simulations, the bonding reaction is simply forbidden in a chosen region on the wall (Fig. 2). The fractional area of the wall surface covered with bonds is denoted by $C$, the bonded coverage. The fractional number of bonds at coverage $C$ with respect to full coverage is defined as $n_{C}=N_{b}(C) / N_{b}(C=1)$. Because crosslinkers above the edge of a nonbond wall region can move a little and bond to an open site, $n_{C}$ tends to be slightly larger than $C$. The ideal fracture stress at coverage $C$ is then

$$
\sigma_{\mathrm{id}}(C)=N_{b}(C) F_{b} / A=n_{C} \sigma_{\mathrm{id}}(C=1) .
$$

Similar to experimental studies [20] and to get a good statistical representation, a set of rectangles on a grid (Fig. 2) were used as the nonbonding region. In the simulations an $8 \times 3$ grid was used. Experimentally, such grids coated with SAMs to prevent bonding can be formed lithographically. However in comparison with such experiments, the simulation nonbonding regions are much smaller, at most $10 \mathrm{~nm}$ on a side. Much smaller debond regions 
are possible experimentally by applying submonolayer coverages of SAMs [20], although the controlled geometry is lost.

\section{E. Tensile data}

Figure 3 shows the tensile stress-strain curves for systems 1-4 at various $C$. The shape of the stress-strain curves is similar for all the systems. The molecular deformations for $C=1$ corresponding to the various stress-strain regimes were described in sec. III A. All the systems fail interfacial as discussed above. The size and coverage effects will now be described.

The $C=1$ stress-strain curve is a master curve for the lower coverage data. At $C<1$ the stress-strain curve follows that for $C=1$ until the failure stress is reached at which point the stress drops to zero. In the tensile mode, this yields two regimes of failure as a function of coverage. For low coverages $(C \leq 0.25)$ failure occurs without any secondary rise in the stress due to bond stretching. At high coverages $(C \geq 0.5)$, bond stretching makes a significant contribution.

The failure stress, $\sigma_{f}$, does not vary with system size. At a given $C$, the shape of the curve is independent of size. In systems 1 and 2 , and for $C=1$ in system 3 , the yield stress is about 4. Otherwise for systems 3 and 4, the yield stress is 3 with a much less pronounced peak. On the other hand, the failure strain, $\varepsilon_{f}$, does depend on the system size. Consider systems 2 and 4 at $C=0$. For system $2, \varepsilon_{f}=0.54$, but for system $4, \varepsilon_{f}=0.20$. For the large system, this $\varepsilon_{f}$ corresponds to the system failing just after the system has reached the yield stress. This is what one expects for surfaces interacting only through LJ interactions.

\section{F. Shear data}

Figure 4 shows the shear mode data for systems 1-4. The yield stress in this case is determined by $C=0$ case which gives $\sigma_{y}=1 u_{0} / d^{3}$ independent of the system size. This stress corresponds to the friction force necessary to slide two surfaces with $\mathrm{LJ}$ interactions 
over each other. The friction stress is well known to be less than the tensile separation stress, since the sliding particles need not fully separate $[22,23]$. For nonzero coverage below the failure strain, the stress rises monotonically with increasing strain. As in the tensile mode, the $C=1$ stress-strain curve is a master curve. The $0<C<1$ curves follow this master curve until failure at which point the stress drops to the yield stress value or sliding friction value.

The failure stress in the shear mode at $C=1$ is the same as in the tensile mode. The failure is also interfacial. Thus, the interfacial strength is the same in either mode. This is not surprising at $C=1$ where the bond forces dominate in determining the interfacial failure stress. The failure stresses are identical for the two modes until the LJ interactions dominate at low $C$ in the tensile mode.

In contrast to the tensile mode, there is no plateau regime in the shear stress-strain curves. Since yielding in shear mode only involves sliding particles over each other, the particles are not separated at the yield strain as in the tensile mode. In the shear mode, the volume is conserved and the stress to deform the whole network increases monotonically beyond the yield strain.

In the shear mode, the deformation sequence is the same as the tensile mode, but the strains are larger due to geometric factors. As shown earlier the failure strain is related to the strain necessary to make taut the minimal paths in the network from the bottom surface to the top surface. This requires a larger strain for the shear mode than for the tensile mode solely due to geometric effects. For an initial wall separation of $L_{z}(0)$ and the same strain, the separation of two points on the top and bottom walls with same $(x, y)$ positions are $(1+\varepsilon) L_{z}(0)$ for tensile mode and $\sqrt{1+\varepsilon^{2}} L_{z}(0)$ for shear mode. For example, at $\dot{\varepsilon}=1$, the length in tensile mode is $2 L_{z}(0)$, but in shear mode, the length is only $\sqrt{2} L_{z}(0)$. Thus, to stretch the minimal paths the same length, the shear simulations must go to larger strains.

As in the tensile mode the failure strain decreases with system size. At $C=0.25$ for system $2, \varepsilon_{f}=1.5$, but for system $4 \varepsilon_{f}=1.0$. For the shear mode, the failure stress predominantly has just one regime. For systems 3 and 4 , a straight line could be fit through 
the $\left(\varepsilon_{f}, \sigma_{f}\right)$ points. This implies a linear relation between $\sigma_{f}$ and $\varepsilon_{f}$ which will be shown in sec. IVA.

\section{DISCUSSION}

\section{A. Failure stress}

While the magnitude of the failure stress is not equal to the ideal fracture stress, the failure stress can still scale as the ideal function. Specifically, let $N_{1}$ and $N_{C}$ be the number of interfacial bonds at full coverage $(C=1)$ and partial coverage, $C$, respectively. Then, if scaling holds, the stress at $C$ is related to the stress at $C=1$ by

$$
\sigma_{C} \sim \frac{N_{C}}{N_{1}} \sigma_{1}=n_{C} \sigma_{1}
$$

The data for the failure stresses (Fig. 5) does in fact exhibit a linear dependence for the shear mode over the complete range of $C$. The linear fit to the shear data is

$$
\sigma_{C}=5.4 n_{C}+0.7 \simeq\left(\sigma_{1}-\sigma_{0}\right) n_{C}+\sigma_{0}, \ldots
$$

where $\sigma_{0}$ is the failure shear stress at $n_{C}=0$. The value of $\sigma_{0}$ is not zero, due to the frictional stress of the sliding surfaces.

As noted above the tensile data has two regimes. At low $n_{C}(<0.3)$, the failure stress is equal to the plateau stress. At larger $n_{C}(\geq 0.5)$, the fit equation is almost identical to that for the shear data,

$$
\sigma_{C}=5.3 n_{C}+0.8
$$

This equation can be written in terms of $\sigma_{p}, \sigma_{1}$, and $n_{C}^{*}$ the crossover point,

$$
\sigma_{C}=\frac{n_{C}-n_{C}^{*}}{1-n_{C}^{*}}\left(\sigma_{1}-\sigma_{p}\right)+\sigma_{p}, n_{C}>n_{C}^{*} .
$$

Equation 10 given $\sigma_{1}=6.2$ and $\sigma_{p}=3.0$ is obtained using $n_{C}^{*}=0.4$; for $n_{C}<n_{C}^{*}, \sigma_{C}=\sigma_{p}$. This equation connects $\sigma_{p}$ and $n_{C}^{*}$ to the y-intercept and slope of Eq. 10. 


\section{B. Failure strain}

For $C=1$ it was shown earlier that the average minimal path determines the failure strain. For $C<1$ the average minimal path does not change much, but the failure strain does. What happens as $C$ decreases from 1 and how does this effect the failure strain? The essential aspect is that the strain becomes inhomogeneous with distinct bulk and interfacial terms.

$$
\varepsilon=\varepsilon_{\text {bulk }}+\varepsilon_{\text {int }}^{\prime}
$$

The fewer the bonds at the interface, the larger the inhomogeneity is.

Since the walls are pulled at constant velocity, a homogeneous strain would yield a linear displacement profile in both the tensile and shear mode. The mechanism for deviations from the linear profile is exhibited in Fig. 6. The figure shows an image of a tensile pull simulation of system 2 at $C=0.1$ and $\varepsilon=0.54$. Tiny, nanometer scale cavities have formed on the bottom surface above the regions which are not chemically bonded to the bottom surface. A few bonds perilously attach the network to the bottom surface. The pull off . occurs at about the same strain as for the completely nonbonded system. In the $C=0.1$ system at $\hat{\varepsilon}=0.54$, the stress has already dropped to $1.0 u_{0} / d^{3}$ from the plateau stress (see Fig. 3(b)). Most of the drop in the stress is due to this pull off as it occurs before the bonds break. The consequence of the interfacial cavities is that the displacement near the bottom surface is larger than the uniform linear profile.

Displacement profiles for this system have been calculated in the following manner. The displacement of each particle at a given $\varepsilon$ relative to $\varepsilon=0$ is calculated. Then these particle displacements are averaged into bins which are layers parallel to the walls. Figure 7 shows the displacement curves for the system of Fig. 6. The first few bins include the wall particles. At the strain of Fig. 6 the displacement field shows a large increase at small $z$ near the bottom surface due to the cavity formation. The increase is due to the cavity formation and produces increased stress on the bonds to the surface. The figure shows the progression of 
this increased interfacial displacement starting from $\varepsilon=0.30$ where the profile is uniform and linear.

In general, the $C=0$ and $C=1$ cases provide boundary values of the failure strain. For $0<C<1$, the nonbonded regions will pull off the bottom surface forming cavities at a strain between the failure strains for $C=0$ and $C=1$. This will yield an excess in strain or strain localization at the interface. The strain localization causes the segments of the minimal paths within the interfacial region to be excessively stretched. In other words, strain localization is the mechanism by which part of a minimal path can become taut without the whole path becoming taut. Bonds within these segments can and do break well before $\varepsilon_{P}$.

Figure 9 shows the failure strains as a function of coverage for system 3 (almost identical to system 4) for tensile and shear modes. The linear fit to the data is

$$
\varepsilon_{f}\left(n_{C}\right)=0.61 n_{C}+0.80
$$

The linear behavior is obeyed for the shear data except at $n_{C}=0$ for which no bonds are broken. Thus, the y-intercept is not equal to the $n_{C}=0$ failure strain. There is insufficient data to study how the failure strain must tend toward $\varepsilon_{f}(0)$ as $n_{C}$ goes to zero. At small $C$ the number of interfacial bonds is small yielding poor statistics.

In the shear mode, cavities cannot form at the surface since the volume is conserved. The strain localization still occurs as shown in Fig. 8. The deviation from the linear profile occurs in a very narrow region at the surface. In shear mode, the interfacial part of the network in the nonbonded regions can slip over the solid surface. Thus, it does not displace as far as the solid surfaces or the interfacially bonded part of the network. Figure 8 shows the average displacement near the bottom wall tends to be closer to 0 than the wall value. In other words, only the part of the network that must move with the wall does; the rest shears as little as it can. This results in a split between bulk and interfacial strain. Then, just like the inhomogeneity in the tensile strain, this gives the mechanism for a constraint on the minimal path segments which forces them to stretch at $\varepsilon<\varepsilon_{P}$. 
For the tensile data in Fig. 9, the linear relation is consistent (albeit for three data points) at $n_{C}>n_{C}^{*}$. For $n_{C}<n_{C}^{*}$, the failure is dominated by van der Waals interactions which yields a different behavior. In tensile, the van der Waals interactions are relatively strong in comparison with the bond interactions. This suggests that the deviation from the failure strain which is based on bonded interactions should occur at a much larger $C$ for tensile than for shear simulations.

\section{Model Modifications}

There are a variety of changes to the model that can be made. Some of these would be basic improvements; others would be to treat the variety of systems available. The system treated here has identical $\mathrm{LJ}$ interaction parameters for all species. It is easy to input differing parameters. Some work has already been done to examine the effect of varying polymer-substrate interaction. Varying the relative preference of the crosslinker and resin to the surface is another important issue to be studied. This directly effects the number of interfacial density of crosslinkers and consequently, the interfacial number of bonds. .

The stiffness of the strands effects the failure strains directly. In the present model, the strands are completely flexible and the extension ratio is large. To make the strands stiffer a bond bending interaction could be added. The form would have to allow bond breaking which most bond bending potentials do not do.

In terms of the minimal paths, bond bending constrains the range of accessible angles. With bond bending, the minimal path may be longer than the failure strain, since bonds could break at angles less than $180^{\circ}$. In this case, the minimal path length may be redefined not to be the contour path, but to be the maximal extension length of the minimal path. The next issue is whether failure could occur at strains even smaller than that corresponding to the maximal extension length. This is equivalent to asking whether the addition of bond bending constraints is sufficient to cause strain inhomogeneities. As discussed above, once inhomogeneity can occur the failure mechanism becomes more local to the inhomogeneous 
region. To answer these issues will require the development and addition of an appropriate bond bending potential.

The number of bonds from the network to the solid surface is the critical determinate of whether failure is cohesive or adhesive. In the present simulations, the crosslinkers bonding to the surface only make a single bond. It is possible for the crosslinkers to make three bonds, but evidently this possibility is low. Thus, to treat system that can cohesively fail, the system would have to be altered such that the bonding probability to the surface increases. One simple means to do this is to increase the density of surface bonding sites; this is equivalent to increasing the wall density. This will have the concomitant effect of strengthening the wall-network LJ interaction which may not be wanted. This can be compensated for by reducing the value of $u_{0}$ in the wall-network LJ interactions. Another possibility is to allow multiple bonds from a crosslinker to a single bond site. This would require strengthening the wall spring constant, since two bonds pulling on the site will be able to pull the wall bead well out of the solid structure. The larger spring constant may require a smaller timestep which is a big price to pay. Treatment of this issue will be done in a future work.

\section{CONCLUSIONS}

The initial results have been presented for coarse-grained, bead-spring molecular dynamics simulations of the fracture of highly crosslinked polymer networks bonded to a solid surface. The correspondence between the stress-strain curve and the sequence of molecular deformations has been detailed. For the present model, bonds are not stretched until strains much larger than the yield strain have been reached. The failure strain for a fully bonded surface is equal to the strain necessary to make taut the average minimal path through the network from the bottom solid surface to the top surface. At bond coverages less than full, nanometer scale cavities form at the surface. This yields an inhomogeneous strain profile with a bulk and an interfacial term. This inhomogeneous strain is an additional constraint on the network which results in segments of the minimal path near the interface becoming 
taut well before the full path. The failure strain is linearly proportional to the number of bonds at the interface. Similarly the failure stress has a linear dependence on the number of interfacial bonds.

In the simulations presented here, failure is always interfacial. The number of bonds at the interface is smaller than in the bulk. This interfacial deficit is an automatic consequence of the network formation of the solid surfaces.

This work was supported by the DOE under contract DE-AC04-94AL8500. Sandia is a multiprogram laboratory operated by Sandia Corp., a Lockheed Martin Company, for the DOE. 


\section{REFERENCES}

[1] Brown, H. Science 1994, 263, 1411.

[2] de Crevoisier, G.; Fabre, P.; Corpart, J.; Leibler, L. Science 1999, 285, 1246.

[3] Henkee, C.; Kramer, E. J. Poly. Sci.: Poly. Phys. Ed. 1984, $22,721$.

[4] Yim, H.; Kent, M.; McNamara, W.; Ivkov, R.; Satija, S.; Majewski, J. Macromol. 1999, 32, 7932 .

[5] Kent, M. S.; Reedy, E. D.; Stevens, M.

[6] Glad, M.; Kramer, E. J. Mats. Sci. 1991, 26, 2273.

[7] Kinloch, A.; Young, R. Fracture Behavior of Polymers. Applied Science Publishers, London, 1983.

[8] Wool, R. Polymer Interfaces: Structure and Strength. Hanser, Munich, 1995.

[9] Termonia, Y. in Computer Simulation of Polymers, Colbourn, E., Ed., (Longman Scientific and Technical, Essex, England). chapter 6, pp 200-227. 1994.

[10] Baljon, A.; Robbins, M. Science 1996, 271, 482.

[11] Binder, K., Ed. Monte Carlo and Molecular Dynamics Simulations in Polymer Science. Oxford, New York, 1995.

[12] Kremer, K.; Grest, G. in Monte Carlo and Molecular Dynamics Simulations in Polymer Science, Binder, K., Ed., (Oxford, New York). chapter 4, pp 194-271. 1995.

[13] de Gennes, P. Scaling Concepts in Polymer Physics. Cornell University, Ithaca, NY, 1979.

[14] Kremer, K.; Grest, G. J. Chem. Phys. 1990, 92, 5057.

[15] Morgan, R.; Kong, F.-M.; Walkup, C. M. Polymer 1984, $25,375$. 
[16] Schneider, J.; Hess, W.; Klein, R. J. Phys. A 1985, 18, 1221.

[17] Hu, T. Combinatorial Algorithms. Addison-Wesley, Reading, 1982.

[18] Thomas, R.; Houston, J.; Crooks, R.; Michalske, T. K. T. J. Am. Chem. Soc. 1995, $117,3830$.

[19] Israelachvili, J. N. Intermolecular and Surface Forces. Academic Press, San Diego, 2nd edition, 1992.

[20] Kent, M. S.; Yim, H.; Matheson, A.; Cogdill, C.; Nelson, G.; Reedy, E. D. Use of self-assembled monolayers to control interface bonding in a model study of interfacial fracture: Pure shear loading. preprint: submitted to J. Adhesion?

[21] The epoxy used in the Sandia experiments (ref. [4]) uses EPON 828 resin and Jeffamine T-403 crosslinker.

[22] Thompson, P. A.; Robbins, M. O. Phys. Rev. A 1990, $\{1,6830$.

[23] Yoshizawa, H.; McGuiggan, P.; Israelachvili, J. Science. 1993, 259, 1305. 


\section{TABLES}

TABLE I. System parameters.

\begin{tabular}{llll}
\hline \hline No. & no. particles & area & height \\
\hline 1 & 14000 & $A_{1}=643$ & 15.1 \\
2 & 51500 & $3 A_{1}=1928$ & 19.9 \\
3 & 91000 & 1928 & 39.2 \\
4 & 170000 & 1928 & 77.0 \\
\hline \hline
\end{tabular}




\section{FIGURES}

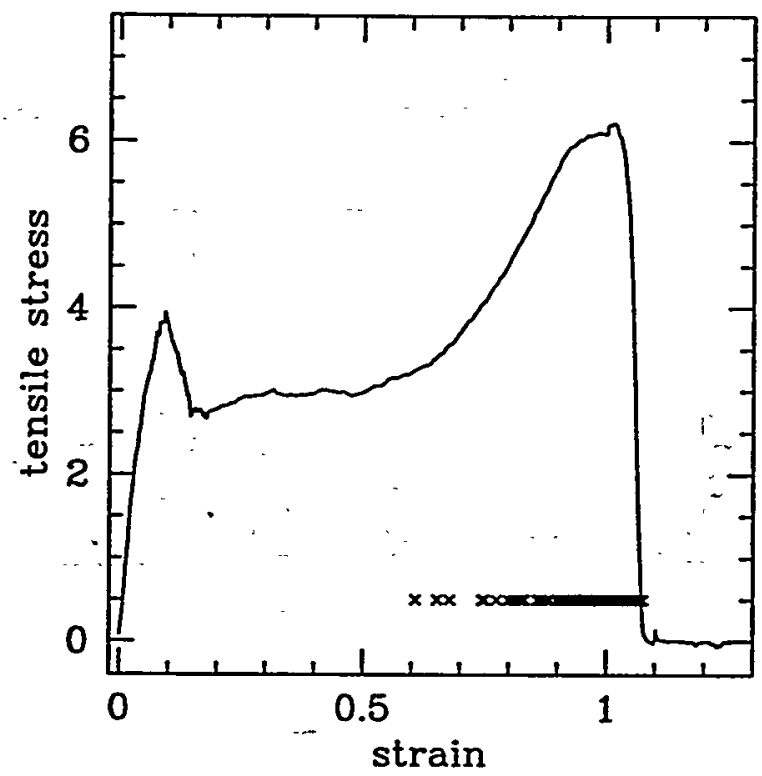

FIG. 1. The tensile stress-strain curve for the fully bonded case of system 2. The points mark the strains at which bond breaking occurs.

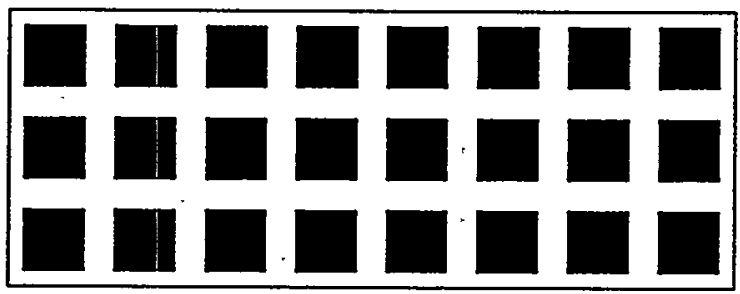

FIG. 2. The checkerboard pattern used to create debond areas of varying size is schematically shown. The black squares represent the debond regions. The size of the squares determine the fraction of debond area. 


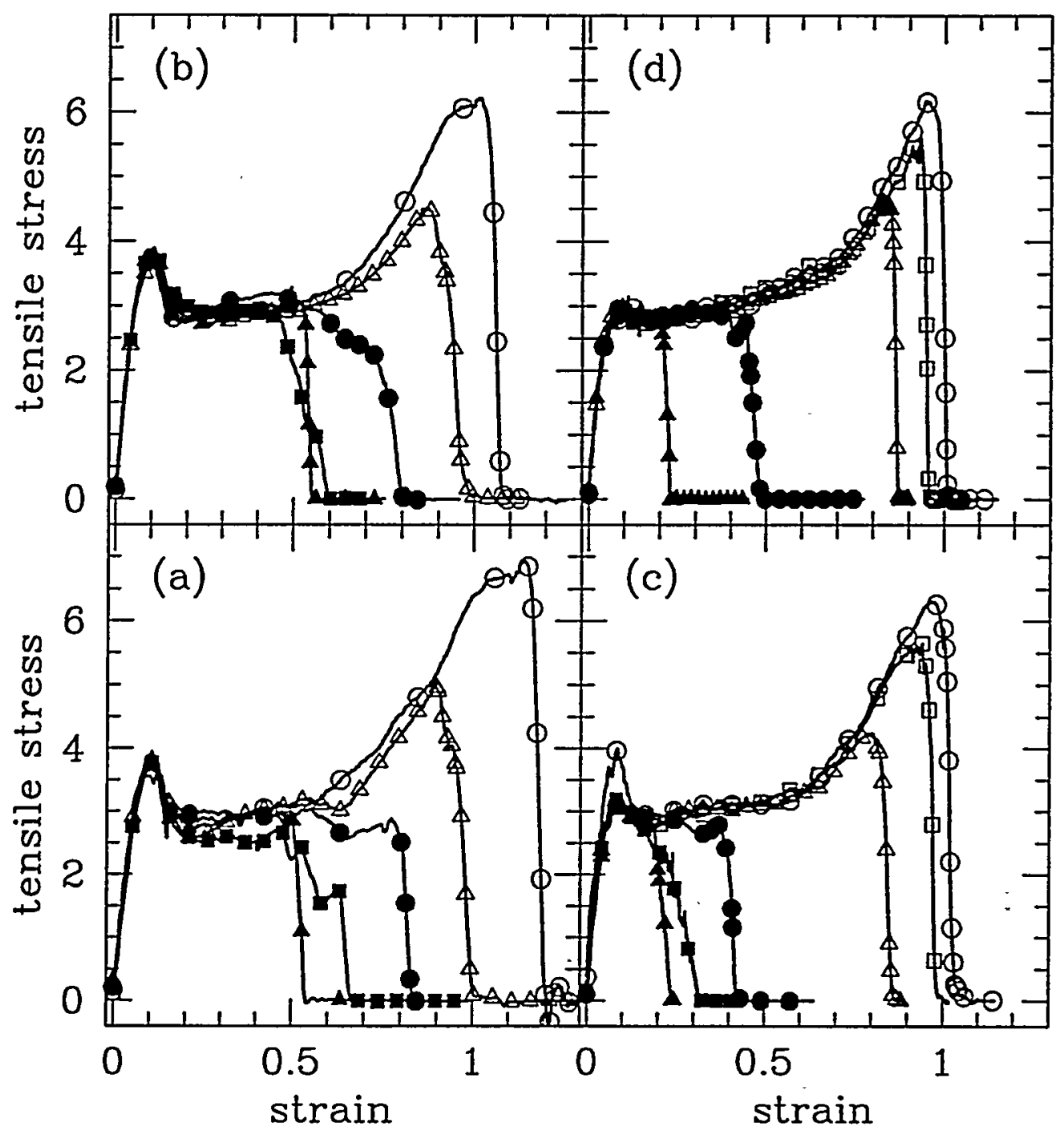

FIG. 3. The tensile stress-strain curves for systems 1 (a), 2 (b), 3 (c) and 4 (d). The point represent different coverages at the bottom surface: $0 \%$ (solid triangle), $10 \%$ (solid square), $25 \%$ (solid circle), $50 \%$, (open triangle), $75 \%$ (open square), $100 \%$ (open circle). 


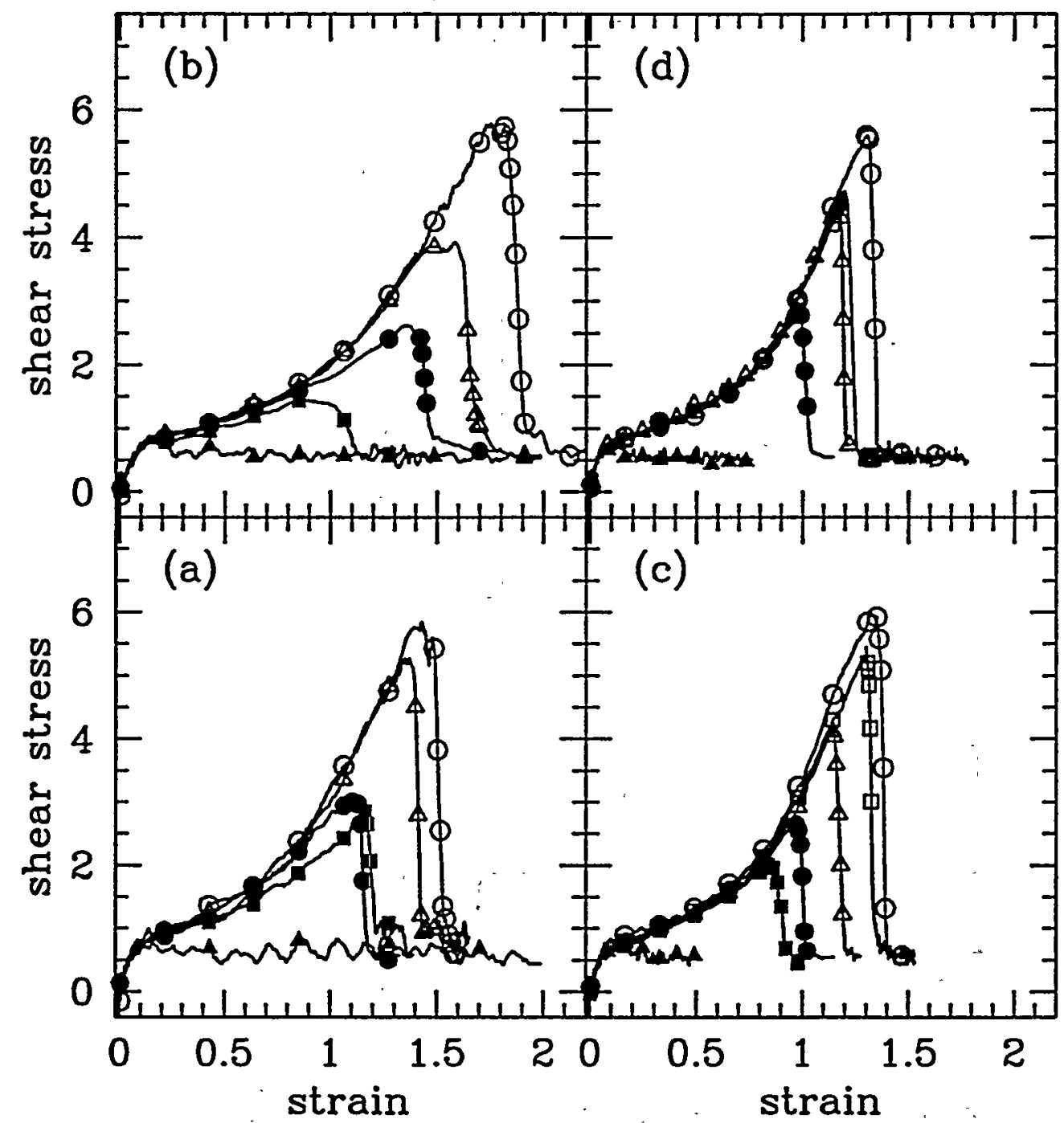

FIG. 4. Shear simulation data. Points are same as Fig. 3. 


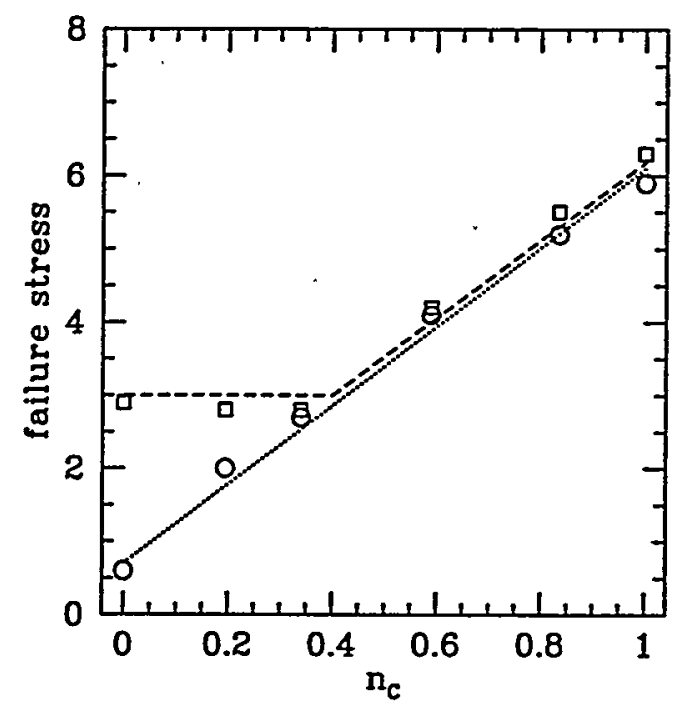

FIG. 5. Failure stress in tensile (squares) and shear (circles) simulations as a function of the fractional number of interfacial bonds.

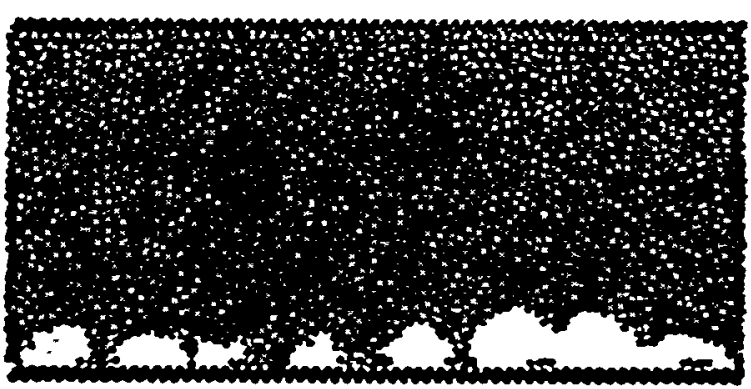

FIG. 6. Image of system 2 at $C=0.1$ under tensile pull at $\varepsilon=0.54$. The pull off above the nonbonded regions at the bottom surface is clearly exhibited.

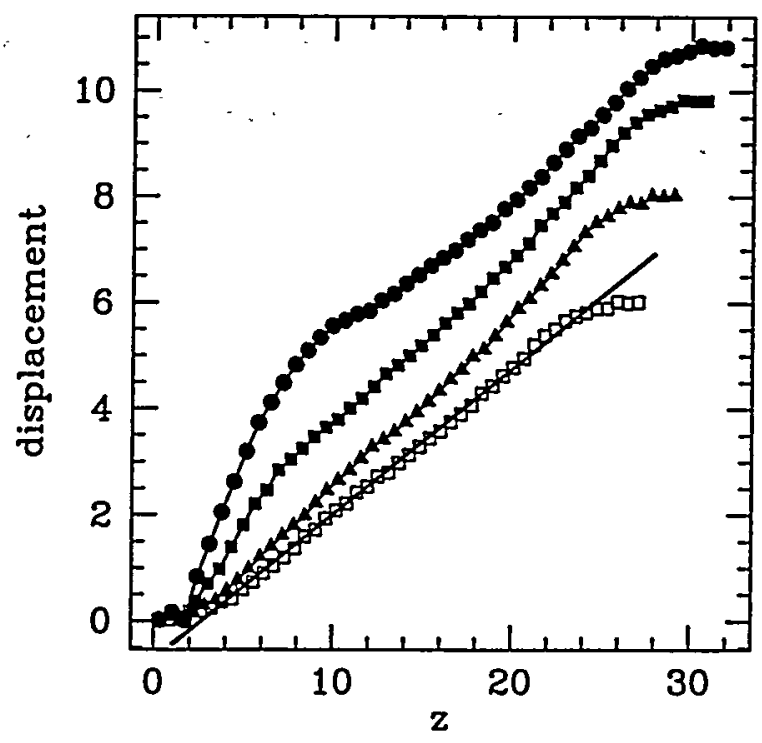


FIG. 7. The displacement profile for system 2 at $C=0.1$ under tensile pull. The data are for the following strains: 0.54 (circle), 0.49 (square), 0.40 (triangle), 0.30 (open square). The circle data is for the strain of Fig. 6. The straight line is a fit to $\varepsilon=0.30$ data excluding the three points on both ends which corresponds to the solid surfaces.

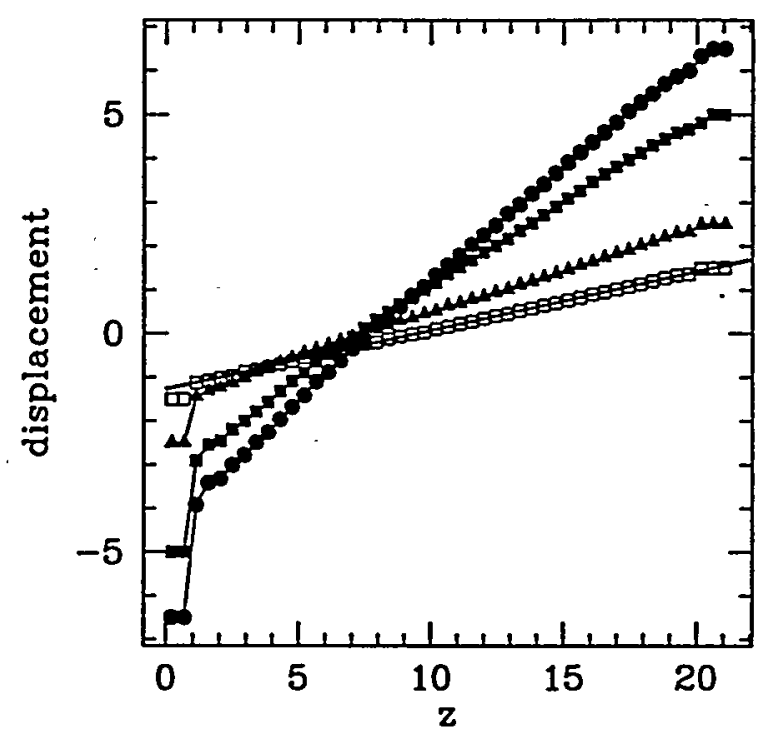

FIG. 8. The displacement profile for system 2 at $C=0.1$ in shear mode. The data are for the following strains: 1.52 (circle), 1.32 (square), 0.99 (triangle), 0.86 (open square). .The straight line is a fit to $\varepsilon=0.86$ data excluding the three points on both ends which corresponds to the solid surfaces.

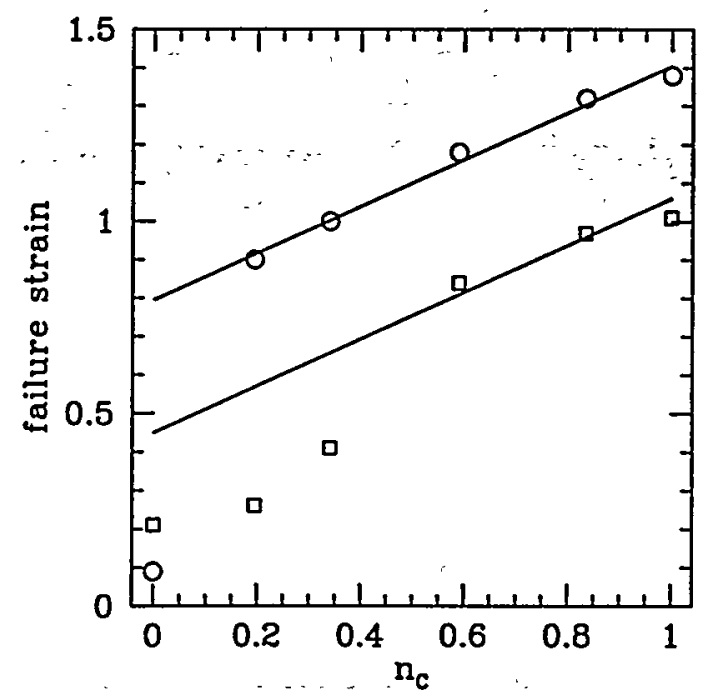


FIG. 9. Failure strain in tensile (squares) and shear (circles) simulations as a function of the fractional number of interfacial bonds. A straight line has been fit to the shear data excluding the $n_{C}=0$ point, and then the same line but different slope is plotted on the tensile data. 